\title{
Gallium Plasmonics: Deep Subwavelength Spectroscopic Imaging of Single and Interacting Gallium Nanoparticles
}

\author{
Mark W. Knight, ${ }^{\dagger}$ Toon Coenen, ${ }^{\dagger}$ Yang Yang, ${ }^{\ddagger}$ Benjamin J. M. Brenny, ${ }^{\dagger}$ Maria Losurdo, ${ }^{\S}$ \\ April S. Brown, ${ }^{\perp}$ Henry O. Everitt, ${ }^{\neq, \perp, l}$ and Albert Polman ${ }^{*,+}$ \\ ${ }^{\dagger}$ Center for Nanophotonics, FOM Institute AMOLF, Science Park Amsterdam 104, 1098 XG Amsterdam, The Netherlands, ${ }^{\ddagger}$ Department of Physics, Duke University, \\ Durham, North Carolina 27708, United States, ${ }^{\S}$ Institute of Inorganic Methodologies and of Plasmas-CNR, and INSTM, via Orabona, 4-70126 Bari, Italy, ${ }^{\perp}$ Department \\ of Electrical and Computer Engineering, Duke University, Durham, North Carolina 27708, United States, and "Army Aviation \& Missile RD\&E Center, RDMR-WDS, \\ Redstone Arsenal, Alabama 35898, United States
}

\begin{abstract}
Gallium has recently been demonstrated as a phasechange plasmonic material offering UV tunability, facile synthesis, and a remarkable stability due to its thin, self-terminating native oxide. However, the dense irregular nanoparticle (NP) ensembles fabricated by molecular-beam epitaxy make optical measurements of individual particles challenging. Here we employ hyperspectral cathodoluminescence $(\mathrm{CL})$ microscopy to characterize the response of single Ga NPs of various sizes within an irregular ensemble by spatially and spectrally resolving both in-plane and out-of-plane

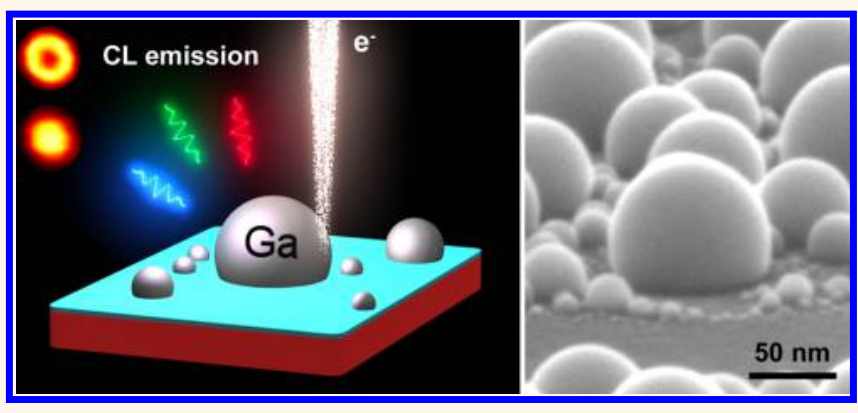
plasmonic modes. These modes, which include hybridized dipolar and higher-order terms due to phase retardation and substrate interactions, are correlated with finite difference time domain (FDTD) electrodynamics calculations that consider the Ga NP contact angle, substrate, and native Ga/Si surface oxidation. This study experimentally confirms previous theoretical predictions of plasmonic size-tunability in single Ga NPs and demonstrates that the plasmonic modes of interacting Ga nanoparticles can hybridize to produce strong hot spots in the ultraviolet. The controlled, robust UV plasmonic resonances of gallium nanoparticles are applicable to energy- and phase-specific applications such as optical memory, environmental remediation, and simultaneous fluorescence and surface-enhanced Raman spectroscopies.
\end{abstract}

KEYWORDS: gallium · cathodoluminescence $\cdot$ nanoparticles $\cdot$ ultraviolet $\cdot$ hyperspectral $\cdot$ plasmonics $\cdot$ dielectric function

$\longrightarrow T^{t}$ allium, a liquid metal near room temperature, has become a key element in both electronic and optoelectronic devices since the start of the microelectronics revolution in the 1960s. ${ }^{1}$ In the past several years, Ga has emerged as a promising new material for plasmonics among a growing family of alternative materials. ${ }^{2,3}$ Among the ultraviolet materials, gallium stands out for its complementary material properties compared with the noble metal nanoparticles (NPs). Unlike noble $\mathrm{Au}$ and seminoble $\mathrm{Ag}, \mathrm{Ga}$ is both environmentally stable and has a Drude-like dielectric function extending from the vacuum ultraviolet through the visible and, in the liquid state, into the infrared spectral region. With a bulk plasma frequency of $14 \mathrm{eV}^{4,5}$ the excitation of bound, coherent oscillations of conduction electrons on the Ga surface, called a localized surface plasmon resonance (LSPR), is possible within the broad bandwidth over which gallium is metallic.

These material properties have become relevant for plasmonic applications because of a range of new, bottom-up fabrication techniques capable of producing pure $\mathrm{Ga}$ NPs. These methods include self-assembly during molecular beam epitaxy (MBE), ${ }^{6,7}$ optically regulated self-assembly, ${ }^{8}$ thermal evaporation, ${ }^{9}$ and colloidal synthesis. ${ }^{10}$ When exposed to atmosphere following synthesis, Ga NPs form a thin, self-terminating native oxide shell that protects the pure metallic core. This $\mathrm{Ga}_{2} \mathrm{O}_{3}$ oxide layer, ${ }^{11}$ which is $0.5-3 \mathrm{~nm}$ thick, ${ }^{10-12}$ provides both structural and chemical stability, allowing the optical response of Ga NPs to remain stable
* Address correspondence to polman@amolf.nl.

Received for review December 18, 2014 and accepted January 28, 2015.

Published online

$10.1021 / \mathrm{nn} 5072254$

C XXXX American Chemical Society 
under atmospheric conditions over many months or years. ${ }^{7,10}$ This stability exceeds other UV-compatible plasmonic materials including silver, which lacks a passivating native oxide, and aluminum. ${ }^{13,14}$

Already, novel applications have been demonstrated that rely on the unique optical and material properties of Ga NPs: UV spectroscopy substrates for simultaneous fluorescence and surface-enhanced Raman spectroscopy (SERS), ${ }^{15}$ highly compact solid-liquid phase change memory elements, ${ }^{16-21}$ phase transition nonlinear substrates, ${ }^{22}$ and graphene/plasmon nanocomposites. ${ }^{23}$ As a first step toward next-generation devices that exploit plasmonic resonance engineering through geometric tuning and hybridization, it is important to demonstrate agreement between the theoretical predictions for gallium and the resonances that exist in fabricated NPs. For noble metal plasmonics, which relies heavily on oxide-free solid Au, the ability to model and predict the response of fabricated structures accurately has been a critical factor enabling the explosive growth of applications demanding an engineered optical response. For $\mathrm{Ga}$, as with the classic plasmonic materials $\mathrm{Au}$ and $\mathrm{Ag}$, the predicted optical properties are based on bulk dielectric functions and classical electrodynamics models. However, validating these predictions for $\mathrm{Ga}$ is especially critical since, unlike most metals, it transitions between solid and liquid phases near room temperature $\left(29.7^{\circ} \mathrm{C}\right)$. To date, obtaining single particle data for $\mathrm{Ga}$ has been complicated by fabrication processes that produced densely packed particle arrays with significant size distributions $>20 \%$. $^{7}$ Within such arrays, standard optical microscopy techniques, such as darkfield spectroscopy, cannot resolve single particles as the interparticle separation lies substantially below the far-field diffraction limit. Recent progress in synthesis has allowed the preparation of colloidal particles with size distributions of $7-8 \%,{ }^{10}$ although inhomogeneous broadening will still affect the resulting ensemble spectra.

In this work we measure the optical response of individual Ga NPs within a densely packed array using hyperspectral cathodoluminescence $(\mathrm{CL})$ microscopy with deeply subwavelength resolution. Using $\mathrm{CL}$ we have nonperturbatively probed the local optical excitability of single particles with diameters between 40-140 nm as a function of both spatial position and frequency. To our knowledge this is the first experimental measurement that both spectrally and spatially resolves the optical response of single $\mathrm{Ga}$ nanoparticles.

\section{RESULTS AND DISCUSSION}

Fabrication and Materials Characterization. Controlled growth of gallium nanoparticle arrays on a silicon substrate was performed using MBE as previously reported (see Methods: Ga NP Fabrication) ${ }^{6,7}$ Briefly, Ga was deposited at room temperature under ultrahigh vacuum (UHV), where the metal self-assembled into liquid nanoparticles through surface diffusion and Ostwald ripening. The optical properties of the $\mathrm{Ga}$ $\mathrm{NPs} / \mathrm{Si}$ ensemble were monitored in real-time by spectroscopic ellipsometry (SE), and growth was terminated when the LSPR of the effective Ga NPs/Si medium reached the designed wavelength.

For an isolated Ga NP within this ensemble four geometric parameters are sufficient to describe both the structure and local dielectric environment: diameter $(D)$, contact angle $(\alpha)$, and the native oxide thicknesses of gallium $\left(T_{\mathrm{GaOx}}\right)$ and silicon $\left(T_{\mathrm{SiOx}}\right)$ (Figure 1A). Both diameter and contact angle can be visualized using high-angle electron micrographs as shown in Figure 1B (see Methods: Structural Characterization). This image also shows the large central Ga NP surrounded by a halo of smaller NPs, a typical feature of Ga NP arrays grown by MBE that is clearly visualized in a normal-incidence SEM image of the sample (Figure 1C). ${ }^{15}$ From this image, we extract a size distribution by fitting each particle with a circle using a generalized Hough transform (Figure 1C, inset). The resulting size distribution $(N=387)$ shows a welldefined peak, and fitting the primary distribution with a Gaussian curve yields a mean diameter of $72 \pm 15 \mathrm{~nm}$. The increasing tail for dimensions below $40 \mathrm{~nm}$ corresponds to the halo of small particles surrounding the larger, isolated nanoparticles.

The dielectric function of pure gallium, corresponding to the core of the $\mathrm{Ga} / \mathrm{Ga}_{2} \mathrm{O}_{3}$ core-shell geometry, was determined using spectroscopic ellipsometry (SE) on smooth Ga films. Briefly, liquid gallium was poured on a glass slide creating a reflective continuous film after which ellipsometric measurements were performed in an inert $\mathrm{N}_{2}$ atmosphere both at liquid and solid Ga temperatures (see Methods: Measuring Permittivity). Our experimentally inferred permittivity for solid Ga, shown in Figure 1D (circles), agrees well with literature values for the dielectric function of $\mathrm{Ga}$ at $300 \mathrm{~K}^{4,5,24,25} \mathrm{~A}$ full comparison spanning the experimental spectral range is provided in Supporting Information, along with downloadable dielectric functions (Figure S1). This detailed comparison was performed in the spirit of previous work on plasmonic materials that has noted considerable discrepancies between published dielectric functions. ${ }^{26}$ While the full band structure of $\mathrm{Ga}$ is complex, the primary features of the solid phase $\mathrm{Ga}$ dielectric function can be attributed to Drude-like free electron oscillations in the blueUV spectral regime, and to interband transitions in the green-red region of the spectrum. ${ }^{2,4,5}$ These interband transitions damp plasmonic behavior beyond the visible $\left(\lambda_{0}>700 \mathrm{~nm}\right)$ based on the well-known SPP resonance condition $\left(\varepsilon_{r}<-2\right)$ for spherical particles. ${ }^{24,27-29}$ In the liquid phase, however, gallium behaves as a pure Drude metal from the UV through the near-infrared and supports plasmonic resonances over a much broader wavelength range (Figure 1D, crosses). 

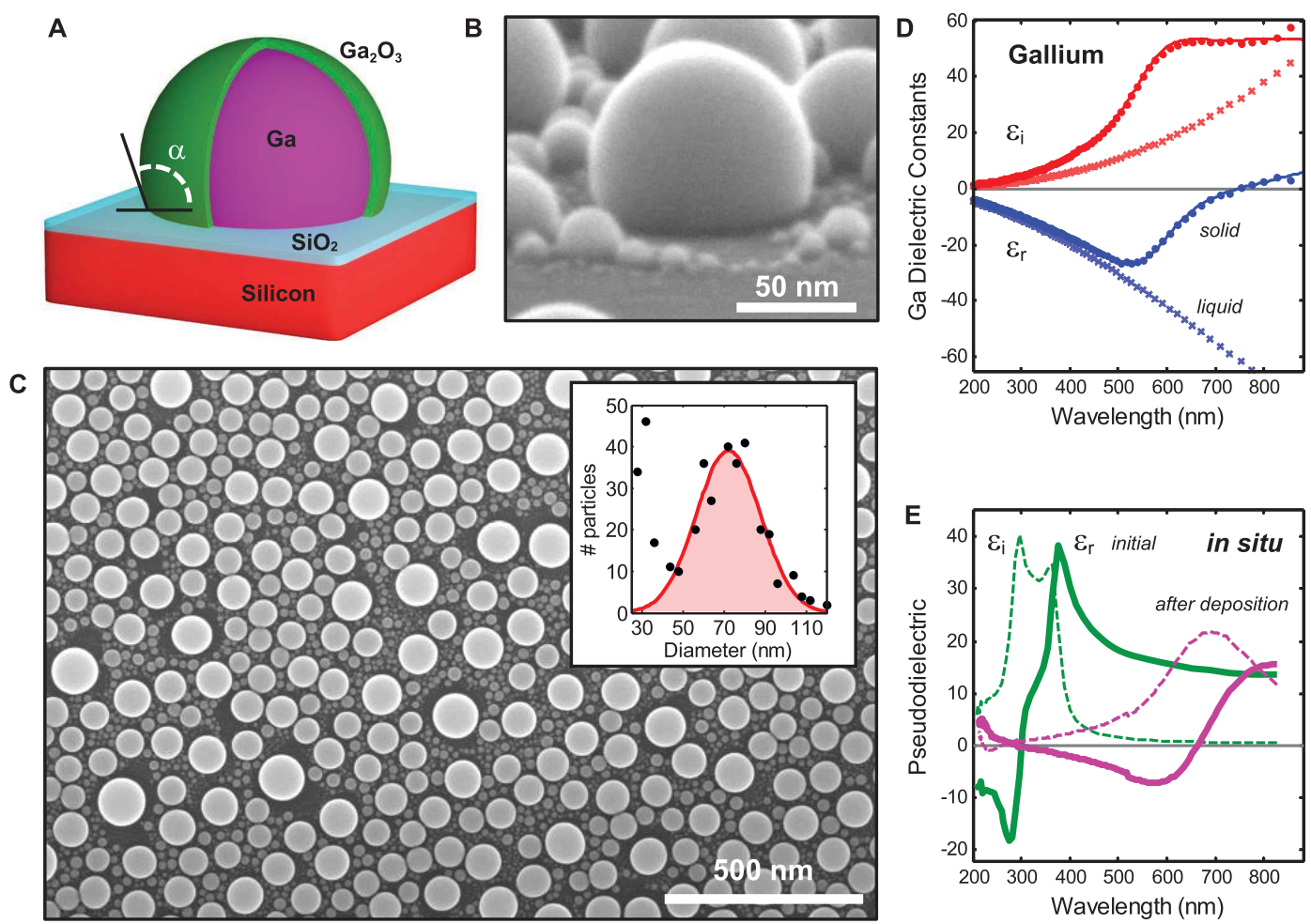

Figure 1. Gallium nanoparticles. (A) The geometry of a gallium nanoparticle can be approximated as a truncated sphere specified by a diameter, contact angle, and oxide thickness. The Si substrate will also have a thin native oxide on the surface. (B) Image of a Ga particle with $D=93 \mathrm{~nm}$ taken at $75^{\circ}$ SEM stage tilt. (C) Typical SEM image of a Ga particle array. Inset: the measured size distribution of particles in this image $(N=387)$ fit with a Gaussian distribution indicating a mean diameter of $72 \pm 15 \mathrm{~nm}$. The significant tail for small particles reflects the large number of small Ga particles formed during the fabrication process. (D) Experimental dielectric functions of solid Ga (circles) and liquid Ga (crosses), and the multicoefficient FDTD fit used in simulations (lines). (E) Pseudodielectric function extracted from ellipsometry measurements on a bare $n$-Si $\langle 111\rangle$ substrate prior to deposition (green), and after Ga particle formation (purple). Real and imaginary components are indicated by solid and dashed lines, respectively.

The ensemble pseudodielectric function, which represents the effective dielectric function of a two phase ambient/sample system (see Methods for details), was measured during deposition in situ by spectroscopic ellipsometry to monitor the nucleation, growth, and optical response of the NP ensemble (Figure 1E), ${ }^{6,30}$ The experimentally measured ellipsometric parameters $\Delta$ and $\psi$ were converted to a pseudodielectric function following the method described previously. ${ }^{6,24}$ The initial pseudodielectric function closely matches the response of crystalline silicon (Figure $1 \mathrm{E}$, green lines). A fit to this initial measurement using a two-layer model $\left(\mathrm{SiO}_{2}\right.$ on crystalline $\left.\mathrm{Si}\right)$, rather than the single layer effective medium model used to calculate the pseudodielectric function, yields a native oxide thickness of $1.1 \pm 0.1 \mathrm{~nm}$. The postdeposition pseudodielectric function, while giving a reproducible metric of the ensemble response, is more complicated to interpret due to the treatment of the nanoparticle/silica/silicon system as a single effective medium (purple lines). While additional modeling can extract effective constituent resonators for some systems, the imaginary component (dashed purple line) can be directly related to the far-field absorption efficiency of the system. ${ }^{6,24,31-33}$
Mapping and Modeling Single Particle Fields. The optical response of the Ga NP ensemble was probed using hyperspectral cathodoluminescence (CL) microscopy, where a complete spectrum of the e-beam induced photoemission was acquired for every pixel in a scan of the sample surface (Figure 2A). To measure CL emission, an energetic beam of $30 \mathrm{keV}$ electrons was focused onto the sample through a small aperture in an Al-coated parabolic mirror. An initial survey scan (Figure 2B) was collected to obtain a high-resolution structural image of the sample and a reference for drift correction during subsequent $\mathrm{CL}$ measurements. During a $\mathrm{CL}$ scan, both secondary electron emission and optical emission were simultaneously captured for each beam position, with the total photoemission from the sample excited at each position collected by a parabolic mirror, then spectrally dispersed and focused onto a CCD sensor array to measure the local excitability spectrum. This process allowed us to construct a hyperspectral "data cube" containing a complete spectrum for every beam position on the sample. The spatial resolution within a data cube is determined by the electron beam diameter (few-nm), the evanescent electric field of the beam $(<20 \mathrm{~nm})$, and the spectral resolution determined by the spectrometer 

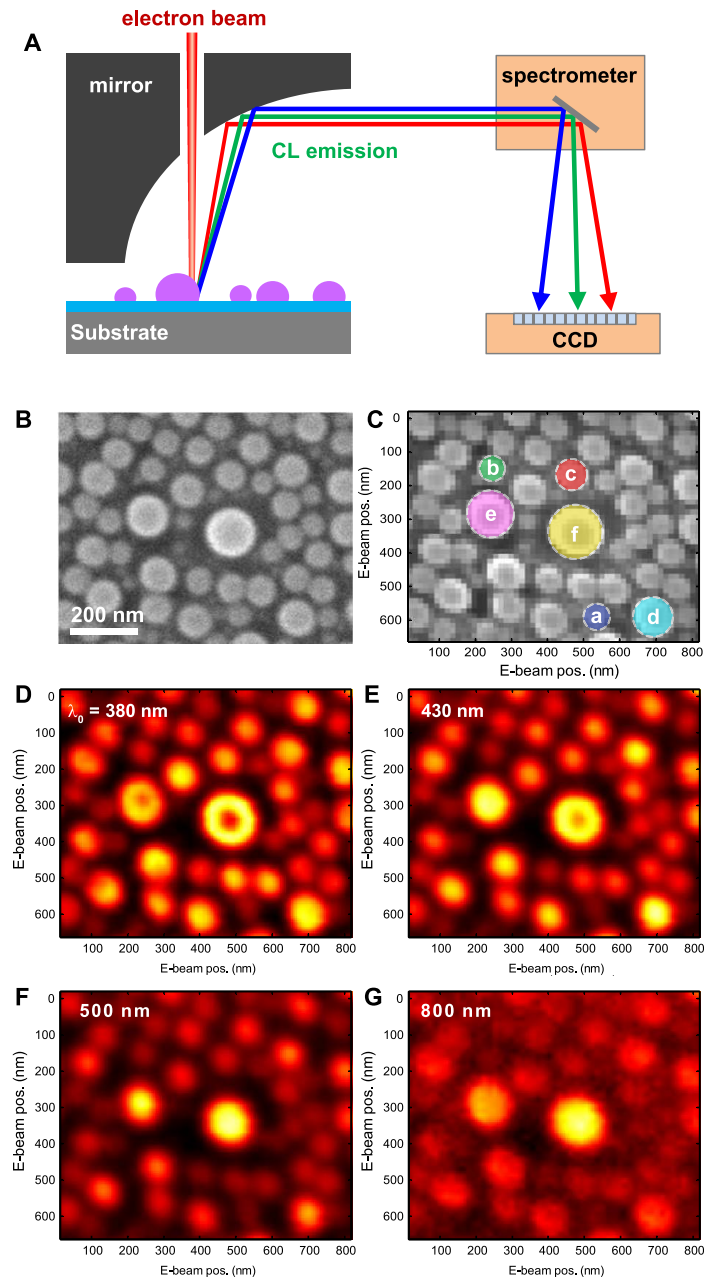

Figure 2. Cathodoluminescence imaging of Ga nanoparticles. (A) Schematic of the hyperspectral cathodoluminescence microscopy system. (B) Survey scan of the sample area acquired prior to $\mathrm{CL}$ imaging. (C) Secondary electron image acquired simultaneously with the $\mathrm{CL}$ scans. Particles used for subsequent analysis are indicated by letters $\mathbf{a}-\mathbf{f}$. From the survey image, the diameters of these particles were estimated to be $\sim 40,60,80,100,120$, and $140 \pm 5 \mathrm{~nm}$. (D-G) CL images at $\lambda_{0}=380,430,500$, and $800 \mathrm{~nm}$. All images were integrated over a spectral bandwidth of $50 \mathrm{~nm}$, normalized to the full color scale, spatially interpolated for clarity in visualizing the CL distributions.

optics (sub-nm). Repeated scans of the same area produced identical optical and structural maps, indicating that gallium nanoparticles are robust against electron-beam induced morphological modification. Details on the implementation of our $\mathrm{CL}$ system are available in Methods: Cathodoluminescence.

From the hyperspectral data a spatial excitability map may be constructed over any spectral region, and that excitability map can be correlated with a corresponding map of the physical sample geometry (Figure $2 \mathrm{C}-\mathrm{G}$ ). Unlike imaging performed with filters, this method allows the selection of an integration bandwidth during postprocessing to balance signalto-noise and mode discrimination optimally. Four representative excitability maps for Ga NP are shown in
Figure 2D-G, with center wavelengths of $\lambda_{0}=380,430$, 500 , and $800 \mathrm{~nm}$ (bandwidth: $50 \mathrm{~nm}$ ). These wavelengths were selected to illustrate the dependence of emission wavelength on excitation location over the range of the spectrometer. It has previously been shown that these excitability maps closely correspond to the z-component of the radiative local density of optical states (LDOS) because the electron beam interacts with the sample through the vertical component of the electric field. ${ }^{27,34-36} \mathrm{~A}$ larger induced local field corresponds to an increased transition probability. ${ }^{27}$ Within the spatial map (Figure $2 \mathrm{C}$ ), six particles are highlighted and assigned the letters $\mathrm{a}-\mathrm{f}$, with corresponding diameters estimated from the survey scan as $D=40,60,80,100,120$, and $140 \mathrm{~nm}$, respectively. This distribution of sizes spans the particle distribution measured in Figure 1C, so analysis of these six NPs can provide a representative understanding of the optical response of this Ga NP ensemble.

These maps show two key features: size-dependent excitability profiles, and a lack of interparticle interactions. At short wavelengths $\left(\lambda_{0}=380,430 \mathrm{~nm}\right)$ there are two distinct excitability distributions: annuli (for $D>100 \mathrm{~nm}$ ), and centered peaks (for $D<100 \mathrm{~nm}$ ). At longer wavelengths $\left(\lambda_{0}=500,800 \mathrm{~nm}\right)$, only weak emission excited near the particle center is observed. For all the particles within these scans, the $\mathrm{CL}$ emission is observed to be symmetric around the particle axis of symmetry. This implies that neighboring particles do not significantly perturb the LDOS of individual particles, allowing us to consider them as if they were isolated Ga NPs on an infinite substrate. ${ }^{37,38}$

This observation supports the inference in previous ellipsometric studies that the ensemble optical response could be well approximated as an amalgam of single-particle responses, ${ }^{6,7,32,33}$ a somewhat surprising finding considering how closely packed the Ga NPs are (Figure 1C). The gap measured between CL emissive particles with $D>40 \mathrm{~nm}$ yields a mean interparticle distance of $25 \mathrm{~nm}$ (Figure S2). Within such an ensemble it has previously been shown that radiative coupling can modify the resonance response of individual scatterers. ${ }^{39}$ While radiative coupling effects may contribute to our signal, the symmetric, consistent $\mathrm{CL}$ measurements between particles of equivalent diameters at different positions within the sample implies that such a contribution contributes at most a minor, perturbative effect. Three factors likely contribute to minimize interparticle coupling between most particles. First, the growth process itself makes the formation of extremely small gaps $(<5 \mathrm{~nm})$ unlikely since two liquid Ga particles that touch during growth will coalesce and form a single large particle. Within our sample fewer than $5 \%$ of all gaps fell within this closely spaced regime. Second, NP contact angles other than $90^{\circ}$ cause a vertical displacement of the NP equators, increasing the nearest neighbor separation beyond 
the gap spacing that may be inferred from the SEM imagery. Third, as shown in Figure 2, the location for maximum $\mathrm{CL}$ excitability in each NP is shifted toward the interior and away from its equatorial rim, which reduces the ability of adjacent particles to interact optically through near-field interaction. There should be some far-field scattering interaction but this has a negligible effect on the single particle response. Combined, these effects allow us to study the response of individual Ga NP within the ensemble and, to a good approximation, treat them as isolated scatterers. A rare exception in which Ga NPs do interact through direct near-field interaction will be discussed shortly.

To understand the CL excitability maps we computationally modeled the response of isolated Ga nanoparticles using experimentally measured dimensions for the particle geometry and by assuming plane wave excitation (Figure 3A, and Methods: Computational Modeling). While plane wave simulations do not directly correspond to $\mathrm{CL}$ maps, they do allow us to visualize the field distributions and energies of the same resonant modes that are experimentally probed using electron beam excitation. For the simulations in Figure 3 , the particle was defined as an oxide-free truncated sphere with $D=140 \mathrm{~nm}$, a contact angle of $\alpha=110^{\circ}$, and a substrate $\mathrm{SiO}_{2}$ layer of $1 \mathrm{~nm}$. The dielectric function of the Ga core was modeled using a multicoefficient fit to the experimental dielectric function of solid Ga. While the phase of the Ga core could not be directly measured during $\mathrm{CL}$ imaging, the $\mathrm{Ga}$ phase actually exerts a relatively small effect on either the resonance position or shape in the Drude-like regime, below the onset of interband transitions (Figure S3). ${ }^{40}$

For a plane wave at normal incidence, an in-plane mode is excited with a dipolar field localized above the equator of the NP (Figure 3A, blue wave). This is shown in Figure $3 \mathrm{~B}$ for a vertical field slice along the polarization axis, illustrating an $E_{z}{ }^{2}$ distribution that matches the annular shape observed in the shortest experimentally accessible $C L$ wavelengths $\left(\lambda_{0}=355-405 \mathrm{~nm}\right)$. This experimental excitability distribution corresponds to the tail of a UV resonance. The field distribution for this resonance, calculated at the peak intensity to show the fundamental mode distribution most cleanly, is shown in Figure $3 C\left(\lambda_{0}=270 \mathrm{~nm}\right)$. Calculated mode profiles at other wavelengths show that this plasmonic mode distribution spans the UV and blue region of the spectrum $\left(\lambda_{0}<400 \mathrm{~nm}\right)$, while the flat bottom of the particle shows many distinct field distributions throughout this range that correspond to a quasicontinuum of narrow band, particle-interface modes that can be viewed as waveguide cavity resonances. ${ }^{41}$ Electric field lines (in white) clearly illustrate the dipolar nature of the in-plane plasmonic resonance. Importantly, the out-of-plane dipolar mode cannot be excited in the quasistatic limit in which the particle is
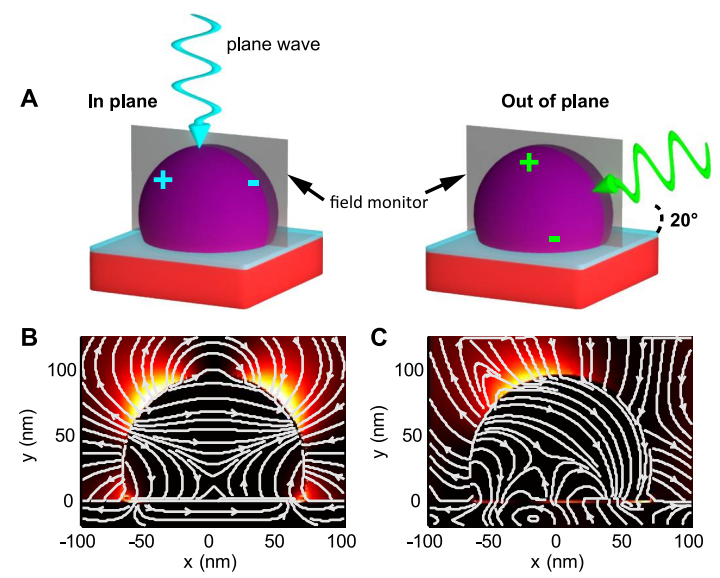

D
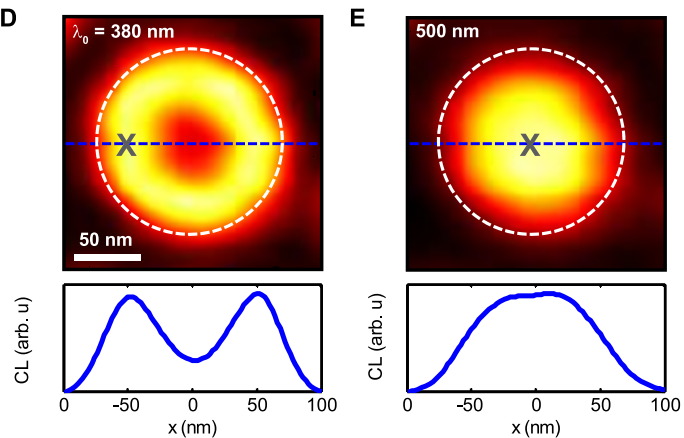

Figure 3. Optical modes of a $D=140 \mathrm{~nm} \mathrm{Ga} \mathrm{NP.} \mathrm{(A)}$ Schematic diagrams of the computational model showing the dipolar charge distributions induced by normal incidence and grazing incidence excitation, and the position of the electric field monitor. $(B, C)$ Calculated field maps of $E_{z}{ }^{2}$, which correspond to the experimental maps, are shown at the calculated peak intensities of these modes for (B) normal incidence excitation with $\lambda_{0}=270 \mathrm{~nm}$ and (C) a p-polarized wave with $\lambda_{0}=470 \mathrm{~nm}$ and at $20^{\circ}$ grazing incidence. Electric field lines are shown in white. (D,E) $C L$ maps of particle $f$ showing a characteristic "annulus" shape for the in-plane modes $\left(\lambda_{0}=380 \mathrm{~nm}\right)$, and a single maximum for the out-of-plane dipolar modes $\left(\lambda_{0}=500 \mathrm{~nm}\right)$. Crosscuts show normalized field intensities across the particle center (cut position: dashed blue lines). Positions marked by $\mathrm{X}$ indicate locations at which nearly pure in-plane or out-ofplane modes may be excited and representative emission spectra for that mode can be measured.

much smaller than the impinging light wavelength. Even for larger particles, including the $140 \mathrm{~nm}$ particle simulated for these field maps, the out-of-plane mode may only be weakly driven via phase retardation across the particle.

To visualize the field distribution of the vertical mode the particle was also simulated using a grazing incidence $p$-polarized plane wave (Figure $3 \mathrm{~A}$, green wave). A calculated $E_{z}{ }^{2}$ field slice at $\lambda_{0}=470 \mathrm{~nm}$ illustrates the resulting vertical dipolar resonance (Figure $3 \mathrm{C}$ ). The asymmetry in the field profile relative to the $\mathrm{CL}$ measurement is due to both the angle of the driving field $\left(20^{\circ}\right)$ and significant phase retardation across the particle due to a diameter $\left(\sim \lambda_{0} / 3.5\right)$ well outside of the quasistatic regime. This asymmetry points to the difference between electron beam and plane wave excitation. While the radiative decay 

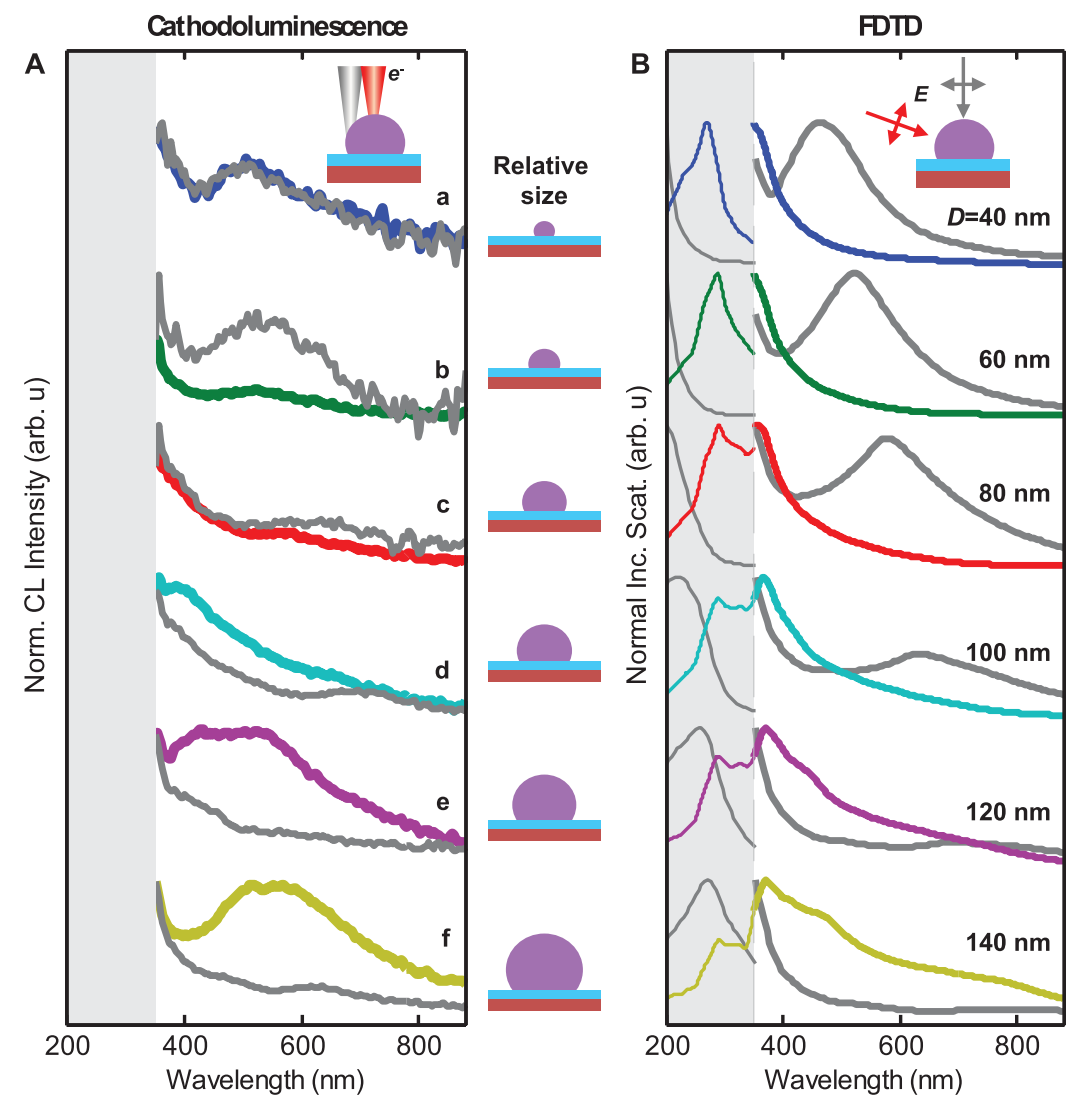

Figure 4. CL spectra of Ga particles. (A) Experimental CL spectra for excitation either at the side of the particle (gray lines) or the center of the particle (colored lines). The shadowed area indicates the spectral region inaccessible to the $\mathrm{CL}$ detector. (B) Spectra calculated for the experimental particle dimensions using FDTD with plane-wave excitation at either normal incidence (gray lines) or grazing incidence $\left(20^{\circ}\right.$, colored lines). All spectra are offset vertically for clarity: thick lines are normalized within the experimental data range, and thin lines are normalized within the $\lambda_{0}=200-350 \mathrm{~nm}$ UV spectral range (shaded region).

(i.e., photon emission) of plasmonic particles does not depend on the excitation source, excitation efficiency does vary for optical and e-beam illumination. Different excitation methods will, therefore, produce different emission spectra. ${ }^{27}$

On the basis of these calculations, the two observed excitability distributions, annuli and bright centers, can be understood as rotationally averaged emission from these in-plane or out-of-plane dipolar resonances (Figure 3D,E). ${ }^{38,42}$ For an in-plane dipole the largest vertical field components will occur at the edges of the NP. It is these vertical field components that couple to the incident electron beam, exciting the in-plane resonance which subsequently decays and emits the photons comprising the $C L$ signal. Since vertically and horizontally polarized emission was collected with equal probability during measurement, this dipolar field distribution exhibits rotational symmetry in the LDOS and forms the "annulus" shape seen in Figure 3D for a $D=140 \mathrm{~nm}$ Ga NP (Figure 2C, particle $f$ ). For an out-of-plane dipole the vertical field component is directly excited and will be maximized at the center, which appears in $\mathrm{CL}$ maps as an emission maximum at the particle center (Figure 3E). For clarity, the particle outline is shown by the dashed white circle, with amplitude crosscuts plotted at the position of the two blue lines.

Single Particle Spectra. On the basis of the LDOS distributions of these two modes we can spectrally isolate the vertical (out-of-plane) and horizontal (inplane) resonances by examining spectra from the particle center and edge, respectively. We will now consider spectra from these positions (indicated by $X$ marks on Figure 3D,E).

For the smallest particle ( $D \approx 40 \mathrm{~nm}$ ) no difference is observed between spectra taken at the particle edge and center (Figure 4A). This is not a physical effect but simply a consequence of the finite spatial resolution of the $\mathrm{CL}$ instrument. For a $30 \mathrm{keV}$ beam the excitability maps have a resolution on the order of $20 \mathrm{~nm} .^{27,36}$ Since the modes are separated by less than half the diameter, the two different resonances cannot be spatially resolved for these smallest particles. Spectra calculated using the experimental geometry of particle $a$, however, clearly show a peak corresponding to normal incidence excitation and an in-plane resonance (Figure 4B). The significant tail on the high-energy side of this peak corresponds to a vertical dipole in the UV (shaded region). While transition radiation (TR), which occurs when a charged particle crosses a 
dielectric interface, could also provide such a spectral feature, ${ }^{16,27}$ we have accounted for this background signal by subtracting the $\mathrm{CL}$ measured within an interstitial region directly adjacent to the measured NP. This removes both TR and incoherent emission from the $\mathrm{Si}$ substrate. A local background subtraction was used rather than a separate, Ga-free area of the substrate since the dense Ga NP ensemble blocks a fraction of TR radiation from the $\mathrm{Si}$ substrate, making the magnitude of the background dependent on the local sample geometry.

For intermediate particles, with $D=60-80 \mathrm{~nm}$, we can resolve distinct spectra for the particle edge and center. The edge spectrum, produced by exciting the in-plane dipolar resonance, redshifts and weakens relative to the tail of the vertical, UV mode. The size tuning observed from $D=40-80 \mathrm{~nm}$ is the result of phase retardation across the particle and is reproduced well in the simulations (Figure 4B). ${ }^{27}$

For the largest particles, with $D>100 \mathrm{~nm}$, the spectra from the edge and center are distinct, and we observe the vertical modes (colored lines) shift from the UV into the detection range of our $\mathrm{CL}$ system $\left(\lambda_{0}>350 \mathrm{~nm}\right)$. Rather than a single resonance, this peak is nearly a continuum of resonances resulting from strong interactions between the Ga NPs and the underlying high index Si substrate, which enables the vertical dipolar mode to hybridize with higher-order modes. ${ }^{33,43-46}$ At the same time, the dipolar in-plane mode (gray lines) has red-shifted out of the plasmonic regime for $\mathrm{Ga}$, and only the tail of a higher-order, multipolar UV resonance is measured (Figure $4 \mathrm{~B}$ ).

The extremely weak $C L$ emission at wavelengths outside of the predicted tunability range of solid Ga $\left(\lambda_{0}>700 \mathrm{~nm}\right)$ may result from a combination of two factors: the Ga may be in the liquid phase as a result of e-beam induced heating during the $\mathrm{CL}$ measurement, ${ }^{16,19}$ or the large and highly polarizable NP may emit due to nonplasmonic geometric resonances similar to those found in dielectric and semiconductor NPs. ${ }^{47,48}$ In the liquid phase the Ga behaves as a Drude metal (Figure 1D) and continues to support plasmonic resonances throughout the near-infrared (NIR). When solid, Ga has a refractive index of $n>5$ in the NIR and may support geometric resonances that are strongly damped by interband absorption.

Although reasonable agreement is observed between the measured and calculated spectra, some discrepancies remain between the observed and calculated resonance energies and relative mode amplitudes. To understand the origin of these differences we have calculated the spectral influence of the geometrical parameters on the in-plane mode: the $\mathrm{Ga}_{2} \mathrm{O}_{3}$ shell thickness, the native $\mathrm{SiO}_{2}$ oxide thickness, and the $\mathrm{Ga}$ contact angle. We find that the Ga oxide shell, which has a refractive index $n_{\mathrm{GaOx}} \approx 2$ and a thickness between $0.5-3.0 \mathrm{~nm},{ }^{10-12,49}$ does not exert a significant influence on the particle spectrum, causing only a negligible redshift (Figure $5 \mathrm{~A}, \mathrm{~B}$ ). By contrast, the in-plane resonance is strongly influenced by both the presence of the native substrate oxide (Figure 5C,D) and variations in the contact angle (Figure 5E,F). Adjusting each parameter over a small, experimentally relevant range significantly affects relative mode amplitudes and positions: both can shift the visible, inplane mode by over $100 \mathrm{~nm}$ in wavelength. On the basis of this sensitivity we conclude that the small differences between the experimental and calculated spectra in Figure 4 are likely due to uncertainty in the particle dimensions extracted from the SEM survey image.

The reason for this sensitivity is the strong interaction between the plasmonic charge oscillations and the high-index silicon substrate. When a plasmonic particle is positioned in vacuum above a dielectric halfspace, the corresponding "image particle" has a surface charge reduced by $(\varepsilon-1) /(\varepsilon+1) \cdot{ }^{28,44,50}$ For silicon, which has a large and strongly dispersive permittivity (Figure 1E, green lines), the substrate interaction can be conceptually understood as the hybridization of a Ga dimer formed by the NP and its image particle with an $\mathrm{SiO}_{2}$-filled nanogap. ${ }^{43,51-53}$ Interactions between real and image particles allow higher order (i.e., nondipolar) modes to couple with the dipolar modes, causing resonances to shift and enabling the excitation of nondipolar charge distributions by the dipolar field of light. ${ }^{46}$ For the normal incidence case shown in Figure 5, increasing either the contact angle or the $\mathrm{SiO}_{2}$ (nanogap) thickness separates and weakens the NP-image interaction. These parameters were computationally tuned to match the experimental spectral peaks of the smallest NPs $(40<D<80 \mathrm{~nm})$, with the best agreement in peak positions found for a simulated $\mathrm{SiO}_{2}$ layer of $1.0 \mathrm{~nm}$ and a contact angle of 110 degrees. Experimentally, the $\mathrm{SiO}_{2}$ thickness was measured to be $1.1 \mathrm{~nm}$ by ellipsometry, and the contact angle was estimated to be between 100 and 130 degrees on the basis of the measured dimensions of angled SEM micrographs and an approximation of the NPs as perfect truncated spheres. Given the sensitivity of the Ga-on-Si system to these geometric parameters, especially the native Si oxide, the agreement between the measured and calculated parameters and mode energies shown in Figure 4 is respectable.

An additional contribution to the experimental and theoretical offsets may originate from differences in the excitation method. The calculations model only the far-field response of the Ga nanoparticle. By contrast, the $C L$ spectra, measured in the far-field, is excited through a near-field interaction between the incident electrons and the local NP fields. It is wellknown that a shift occurs in the spectral response when transitioning between the near- and far-field regimes. $^{54,55}$ 

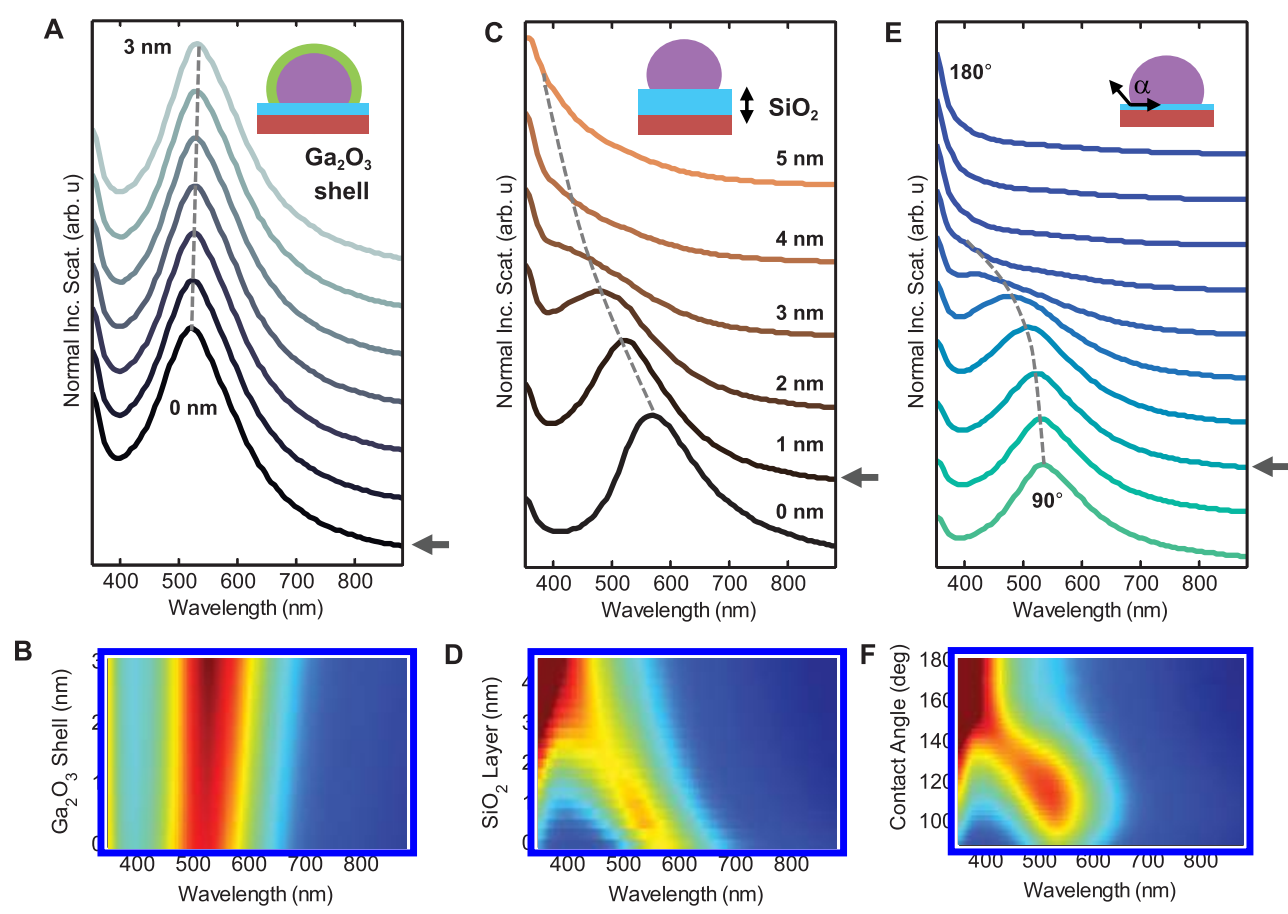

Figure 5. Spectral influence of Ga particle parameters: oxide shell thickness, substrate oxide thickness, and contact angle. Spectra were calculated for a $D=60 \mathrm{~nm}$ Ga particle using normal incidence excitation. (A,B) Effect of the oxide shell thickness, calculated for $0 \mathrm{~nm}$ (unoxidized) to $3 \mathrm{~nm}$ with a $1 \mathrm{~nm}$ layer of $\mathrm{SiO}_{2}$ on the $\mathrm{Si}$ substrate and a $110^{\circ}$ contact angle. $(C, D)$ Effect of the native oxide layer on the $\mathrm{Si}$ substrate, calculated for $0-5 \mathrm{~nm}$, no $\mathrm{Ga}_{2} \mathrm{O}_{3}$ shell, and a $110^{\circ}$ contact angle. (E,F) Influence of contact angle, calculated for angles between $90^{\circ}$ (a hemisphere) and $180^{\circ}$ (a perfect sphere). The substrate oxide was set at $1 \mathrm{~nm}$, with $\mathrm{no} \mathrm{Ga}_{2} \mathrm{O}_{3}$ shell. Dashed gray lines are provided as a guide to the eye. Gray arrows indicate the parameters used to calculate the NP resonances shown in Figures 3 and 4.

Interacting Ga Nanoparticles. While MBE fabrication generally produces Ga NP ensembles without significant near-field interactions between particles (as shown in Figure 2), a small number of closely spaced Ga NPs do form strongly interacting clusters that can hybridize and form new resonant modes. ${ }^{43,53,56}$ The interaction between these clusters can be significantly influenced by both the relative particle diameters and the contact angle, which will add a vertical offset between the particle equators and in-plane dipolar modes of different sized particles. While the gap spacings were below the $\sim 10 \mathrm{~nm}$ resolution of our survey image (Figure $6 \mathrm{~A}$ ), a $\mathrm{CL}$ scan within this region (dashed white box) allows us to visualize the modification of the LDOS within interacting clusters compared to the isolated particle case for four nanoparticles with different diameters (Figure 6B). It is important to note that since $\mathrm{CL}$ measures only the vertical field component, field enhancements in interparticle "hot spots" appear in $\mathrm{CL}$ as dark gaps bounded by bright particle edges. $^{57}$

At short wavelengths, a strong spatial asymmetry in the $\mathrm{CL}$ map is observed: the smaller satellite particles appear as asymmetric dipoles (Figure 6C,D), while the bright "hot spot" adjacent to the central particle is observed to be wavelength-dependent. For the leftmost satellite particle, this hot spot is strongest near the UV edge of our CL detection range, while the rightmost particle exhibits a maximum at $430 \mathrm{~nm}$ because it has both a larger diameter and a larger gap. For longer wavelengths, where the vertical mode dominates and dipolar charge oscillations are spatially well separated, we observe a substantial reduction in interparticle coupling (Figure 6E,F). These trends are reproduced well by FDTD calculations that approximate the cluster geometry using diameters from the survey image and interparticle gaps of $5 \mathrm{~nm}$ (Figure 6G). For this geometry, the normalized, polarization-averaged $\left|E_{z}\right|^{2}$ reproduces the experimental observations of coupling-induced LDOS asymmetries at short wavelengths $\left(\lambda_{0}=380 \mathrm{~nm}\right.$, Figure $6 \mathrm{H})$ and dielectric screening of the electric field at long wavelengths where $\varepsilon_{r}>-2$ (i.e., $\lambda_{0}=800 \mathrm{~nm}$ in Figure 6l).

While strong interparticle coupling occurred infrequently on our sample, engineered arrays of hybridized plasmonic Ga clusters may be important for applications that rely on large electric fields, such as surface enhanced Raman scattering (SERS) which scales as approximately the fourth power of the local field. ${ }^{58-61}$ Previous work demonstrating SERS spectroscopy on Ga NP substrates hypothesized that significant enhancements in MBE fabricated substrates occurred between coupled asymmetric dimers, ${ }^{7,15}$ a hypothesis supported by Mueller matrix ellipsometry measurements showing nonspecular scattering. ${ }^{32}$ The $\mathrm{CL}$ measurements shown here are the first direct evidence that this type of asymmetric interparticle coupling occurs in Ga NP ensembles. 
A
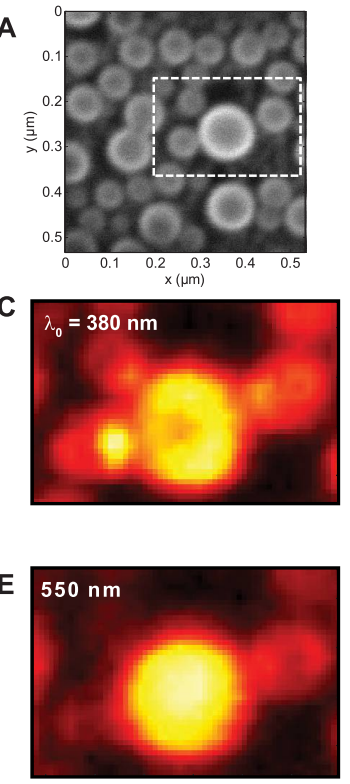

B
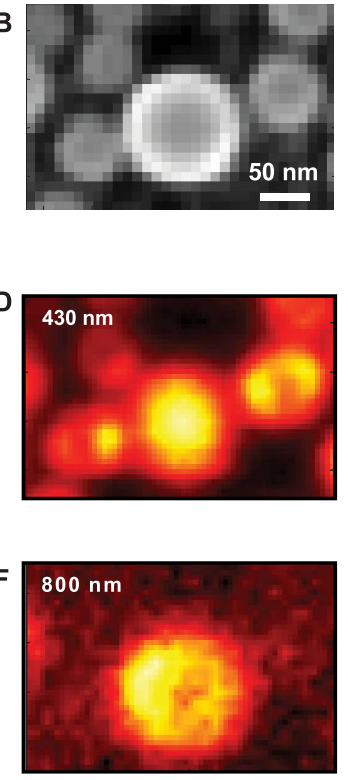

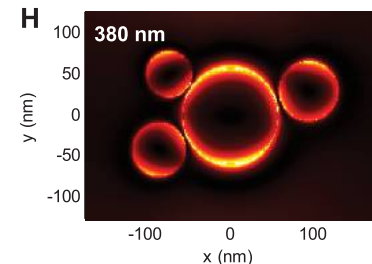

FDTD
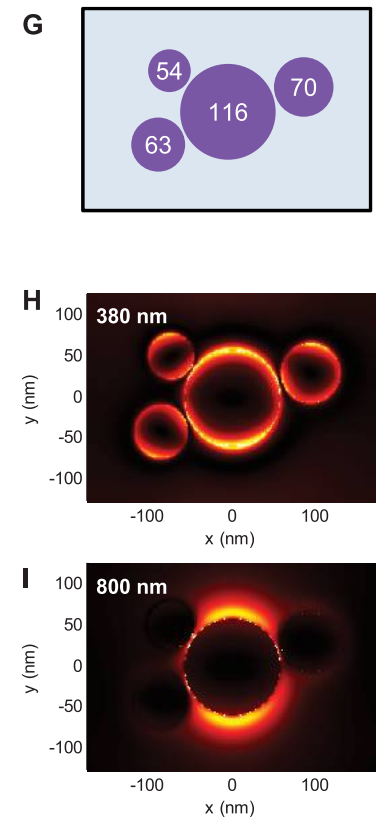

Figure 6. Hybridization in Ga plasmons. (A) Survey image with the scan area indicated by the white dashed box. (B) Simultaneously acquired secondary electron image of the Ga NP cluster. (C-F) CL maps of the cluster at $\lambda_{0}=380,430,550$, and $800 \mathrm{~nm}$, with an integration bandwidth of $50 \mathrm{~nm}$. (G) Simulation geometry, with particle diameters indicated. (H,I) Simulated in-plane field maps showing polarization-averaged $E_{z}^{2}$. Asymmetries in the field profiles correspond with the $C L$ maps and indicate interparticle interaction.

It is important to note that, in contrast to the noble metal substrates commonly exploited for SERS spectroscopy, gallium NPs absorb strongly within the plasmonic regime. The resulting heating of the NPs and surrounding environment, combined with the high energy of the SPPs, can either thermally or chemically alter the analyte. ${ }^{62}$ This effect offers the potential for selfmonitoring of environmental remediation efforts by simultaneous SERS and fluorescence spectroscopies. ${ }^{15}$

\section{CONCLUSION}

Using hyperspectral cathodoluminescence imaging we have conducted deeply subwavelength studies of isolated and coupled Ga NP plasmon modes. Individual nanoparticles were observed to exhibit both size tuning and frequency-dependent spatial LDOS profiles. These observed modes were correlated with electrodynamic calculations of the plasmonic mode profiles and the backscattered emission resulting from plasmon decay using experimentally measured $\mathrm{Ga}$ dielectric functions for both solid and liquid-phase gallium. At blue and ultraviolet wavelengths we have found that the response of these Ga particles is dominated by an in-plane dipolar oscillation with a position and amplitude strongly influenced by the Si substrate. The long-wavelength response, by contrast, is almost purely determined by a vertical dipolar resonance. We have also offered the first experimental observation of direct $\mathrm{Ga}$ interparticle coupling, with measurements of wavelength-dependent hotspots between a large central particle and proximal satellite particles. The high resolution, single particle $\mathrm{CL}$ measurements shown here show reasonable agreement with planewave modeling based on our experimentally measured dielectric functions. Combined, this study provides a first understanding of single Ga NP resonances from 200 to $880 \mathrm{~nm}$, spanning the UV through the NIR spectral regime.

\section{METHODS}

Ga NP Fabrication. Gallium nanoparticle ensembles were grown by molecular beam epitaxy (MBE) of pure Ga on a $2^{\prime \prime}$ wafer of n-type, $\langle 111\rangle$ Si with only a thin native oxide. After a preliminary degas of the $\mathrm{Si}$ sample in the MBE load-lock chamber at $200{ }^{\circ} \mathrm{C}$, the $\mathrm{Si}$ substrate had a residual $\mathrm{SiO}_{2}$ native oxide thickness of $1.1 \pm 0.1 \mathrm{~nm}$. The MBE chamber was maintained under ultrahigh vacuum $\left(10^{-11}\right.$ Torr) during deposition. NP growth took $263 \mathrm{~s}$ at $300 \mathrm{~K}$ resulting in Ga NPs in a liquid state. ${ }^{10,16}$ During deposition the Ga film self-assembled into nanoparticles through a process of adsorption, surface diffusion, and coalescence through Ostwald ripening. ${ }^{31,63}$ Real-time monitoring of this process was performed by in situ spectroscopic ellipsometry (SE) from $\lambda_{0}=200-820 \mathrm{~nm}$ at a $20^{\circ}$ grazing angle of incidence, permitting the termination of growth when the desired optical properties were attained (Figure 1E). ${ }^{6,30}$ Samples were stored in ambient atmosphere for 21 months prior to the $\mathrm{CL}$ measurements, demonstrating the robustness of the native oxide shell.

Structural Characterization. Imaging of individual Ga nanoparticles to measure geometry was performed using a FEG SEM (FEI Verios) with an accelerating voltage of $10 \mathrm{kV}$ and a specimen 
current of $4.9 \mathrm{pA}$. Secondary electron emission was measured using an in-column scintillation detector in combination with an immersion lens. To permit observation of the contact angle the sample was tilted at $75^{\circ}$.

Cathodoluminescence. $\mathrm{CL}$ imaging was performed using a modified scanning electron microscope (SEM, FEI XL-30). A $30 \mathrm{keV}, 800 \mathrm{pA}$ beam of electrons was focused through an Al half-paraboloidal mirror onto a sample with a few-nm spot size. The mirror focus was precisely aligned to overlap the e-beam impact point, using a custom piezoelectric micromanipulation stage, and the resulting optical emission from the sample was directed to an optical bench attached to the outside of the SEM chamber. The collimated light was then either imaged onto a $1024 \times 1024$ Si CCD to ensure proper alignment, or it was fibercoupled into a spectrometer for analysis. Hyperspectral CL maps were acquired by raster-scanning the e-beam across the sample and recording a raw spectrum for each beam position (per-pixel integration time: $250 \mathrm{~ms}$ ). Spectroscopy was done using a grating spectrometer (grating: $500 \mathrm{~nm}$ blaze, $150 \mathrm{~g} / \mathrm{mm}$ ) with a Si CCD (Princeton Instruments: back-illuminated SPEC-10). Separately, the overall system response was ascertained by dividing the theoretical transition radiation (TR) spectrum from aluminum by a measured $C L$ emission spectrum from a single aluminum crystal, where the absence of surface texturing (translational symmetry) prevented the emission of SPPs and only TR was emitted. ${ }^{64}$ The raw gallium spectra were multiplied by this correction factor to account for the properties of the measurement system.

Computational Modeling. The optical response of Ga NPs was modeled using a commercial implementation of the finitedifference time-domain method (FDTD, Lumerical). ${ }^{65}$ The simulated NP geometry was specified as an isolated, truncated sphere of diameter $D$ forming a contact angle $\alpha$ with an infinite substrate. The material properties of $\mathrm{Ga}$ and $\mathrm{Si}$ were modeled using broadband multicoefficient fits to experimental dielectric values. $^{24,66}$ The measured Ga dielectric function used in this work, along with literature values, are compared in the Supporting Information and made available for download. ${ }^{2,5,24}$ The $\mathrm{Ga}_{2} \mathrm{O}_{3}$ shell was approximated using the Cauchy dispersion model fit of Rebien et al.: $n=n_{\infty}+B / \lambda^{2}+C / \lambda^{4}$, with $n_{\infty}=1.891$, $B=0.0110 \mu \mathrm{m}^{2}$, and $C=0.00048 \mu \mathrm{m}^{4}$ (with $\lambda$ in $\left.\mu \mathrm{m}\right) .{ }^{49}$ Particle properties were calculated using the total-field scattered-field (TFSF) formulation where, by separating the simulation domain into regions with either the complete field (at the particle) or only the scattered field (far from the particle), the total power scattered into the far-field by the particle can be calculated. Only power outflow into the upper half-space was included in the integrated spectra to facilitate comparison with the hyperspectral CL measurements. For normal incidence planewave excitation, spectra and field distributions were calculated using a broadband source. For off-normal excitation, where the source angle in FDTD exhibits frequency dependence, individual spectral points were calculated every $10 \mathrm{~nm}$ to permit a well-defined grazing incidence angle $\left(20^{\circ}\right)$ capable of driving out-of-plane modes. ${ }^{33}$ The simulation space was discretized using a mesh step of $1 \mathrm{~nm}$ within the particle region with a refined mesh dimension of $0.25 \mathrm{~nm}$ within the thin oxide layers of both the Ga NP and substrate. Far from the particle, a graded mesh approach increased the mesh step to $4 \mathrm{~nm}$ to reduce the computational cost of the simulations without sacrificing accuracy.

Dielectric Function Measurement. Pure gallium ingots (99.9999\% pure) were purchased from GoodFellow. The Ga processing was performed in a glovebox purged with $\mathrm{N}_{2}$ to avoid oxygen atmosphere. Warming the vial liquefied the $\mathrm{Ga}$, which was then poured onto a glass slide at $308 \mathrm{~K}$. The Ga homogeneously wet the glass, forming a smooth, reflective liquid layer. The liquid sample was then transferred to a variable angle spectroscopic UVISEL (Horiba Jobin Yvon) in a continuously purged measurement cell to avoid any atmospheric adsorption or oxidation of the liquid film. Ellipsometric spectra were acquired in the range $190-1650 \mathrm{~nm}$ to obtain the dielectric function of liquid gallium. In the cell, the sample is mounted on a temperature-controlled holder that was set to a temperature of $278 \mathrm{~K}$ to solidify the $\mathrm{Ga}$ film. Subsequently, the ellipsometric spectrum was acquired again to obtain the dielectric function of solid Ga.

The optical constants of gallium were extracted from the measured data using a point-by-point fit, assuming a two layer (Ga-surface/Ga-bulk) model with bulk Ga (5 $\mu \mathrm{m}$ thick) and the rough surface layer modeled using the Bruggeman effective medium approximation (50\% Ga, 50\% voids). ${ }^{67}$

After ellipsometric measurements the thickness of the solid film was estimated to be $\sim 5 \mu \mathrm{m}$, both by microbalance and surface profilometry (Alpha-Step). These methods were used to estimate film thickness following optical characterization since the Ga film was significantly thicker than the penetration depth of light. The solid Ga film was also measured by atomic force microscopy and found to have $1.1 \mathrm{~nm}$ root-mean-square (RMS) surface roughness.

The extracted dielectric functions for liquid and solid $\mathrm{Ga}$ are plotted in Figure 1D, with a comparison to literature dielectric functions in Figure S1. This comparison shows good agreement between the measured dielectric function for solid $\mathrm{Ga}$ and previous literature values.

Conflict of Interest: The authors declare the following competing financial interest(s): A.P. is co-founder and co-owner of Delmic BV, a startup company that has developed a commercial product based on the cathodoluminescence system that was used in this work.

Acknowledgment. The authors thank N. J. Halas for her support during the early phases of this project and acknowledge the support of the Army's Competitive In-House Laboratory Innovative Research program, the Office of Naval Research, and the Katherine Goodman Stern fellowship program. This work is part of the research program of the Foundation for Fundamental Research on Matter (FOM), which is part of The Netherlands Organization for Scientific Research (NWO), and NanoNextNL, a nanotechnology program funded by the Dutch ministry of economic affairs and the European Research Counsel. M.L. acknowledges the National Research Council (CNR) for financial support from the STM2014 program. The authors also acknowledge the SARA Computing and Networking Services (www.sara.nl) for their support in using the Lisa Computer Cluster.

Supporting Information Available: Experimental dielectric functions for solid and liquid phase gallium, a characterization of interparticle gaps, and the influence of Ga phase on spectra. This material is available free of charge via the Internet at http://pubs.acs.org.

\section{REFERENCES AND NOTES}

1. Moskalyk, R. R. Gallium: The Backbone of the Electronics Industry. Miner. Eng. 2003, 16, 921-929.

2. McMahon, J. M.; Schatz, G. C.; Gray, S. K. Plasmonics in the Ultraviolet with the Poor Metals Al, Ga, In, Sn, Ti, Pb, and Bi. Phys. Chem. Chem. Phys. 2013, 15, 5415-5423.

3. Naik, G. V.; Shalaev, V. M.; Boltasseva, A. Alternative Plasmonic Materials: Beyond Gold and Silver. Adv. Mater. 2013, 25, 3264-3294.

4. Hunderi, O.; Ryberg, R. Band Structure and Optical Properties of Gallium. J. Phys. F: Met. Phys. 1974, 4, 2084-2095.

5. Jezequel, G.; Lemonnier, J. C.; Thomas, J. Optical Properties of Gallium Films between 2 and $15 \mathrm{eV}$. J. Phys. F: Met. Phys. 1977, 7, 1613-1622.

6. Wu, P. C.; Kim, T.-H.; Brown, A. S.; Losurdo, M.; Bruno, G.; Everitt, H. O. Real-Time Plasmon Resonance Tuning of Liquid Ga Nanoparticles by In Situ Spectroscopic Ellipsometry. Appl. Phys. Lett. 2007, 90, 103119.

7. Wu, P. C.; Khoury, C. G.; Kim, T.-H.; Yang, Y.; Losurdo, M.; Bianco, G. V.; Vo-Dinh, T.; Brown, A. S.; Everitt, H. O. Demonstration of Surface-Enhanced Raman Scattering by Tunable, Plasmonic Gallium Nanoparticles. J. Am. Chem. Soc. 2009, 131, 12032-12033.

8. MacDonald, K. F.; Fedotov, V. A.; Pochon, S.; Ross, K. J.; Stevens, G. C.; Zheludev, N. I.; Brocklesby, W. S.; Emel'yanov, 
V. I. Optical Control of Gallium Nanoparticle Growth. Appl. Phys. Lett. 2002, 80, 1643-1645.

9. Ghigna, P.; Spinolo, G.; Parravicini, G. B.; Stella, A.; Migliori, A.; Kofman, R. Metallic versus Covalent Bonding: Ga Nanoparticles as a Case Study. J. Am. Chem. Soc. 2007, 129, 8026-8033.

10. Yarema, M.; Wörle, M.; Rossell, M. D.; Erni, R.; Caputo, R.; Protesescu, L.; Kravchyk, K. V.; Dirin, D. N.; Lienau, K.; von Rohr, F.; et al. Monodisperse Colloidal Gallium Nanoparticles: Synthesis, Low Temperature Crystallization, Surface Plasmon Resonance and Li-lon Storage. J. Am. Chem. Soc. 2014, 136, 12422-12430.

11. Schön, G. Auger and Direct Electron Spectra in X-Ray Photoelectron Studies of Zinc, Zinc Oxide, Gallium and Gallium Oxide. J. Electron Spectrosc. Relat. Phenom. 1973, 2, 75-86.

12. Regan, M. J.; Tostmann, H.; Pershan, P. S.; Magnussen, O. M.; DiMasi, E.; Ocko, B. M.; Deutsch, M. X-Ray Study of the Oxidation of Liquid-Gallium Surfaces. Phys. Rev. B: Condens. Matter Mater. Phys. 1997, 55, 10786-10790.

13. Langhammer, C.; Schwind, M.; Kasemo, B.; Zoric, I. Localized Surface Plasmon Resonances in Aluminum Nanodisks. Nano Lett. 2008, 8, 1461-1471.

14. Szklarska-Smialowska, Z. Pitting Corrosion of Aluminum. Corros. Sci. 1999, 41, 1743-1767.

15. Yang, Y.; Callahan, J. M.; Kim, T.-H.; Brown, A. S.; Everitt, H. O. Ultraviolet Nanoplasmonics: A Demonstration of SurfaceEnhanced Raman Spectroscopy, Fluorescence, and Photodegradation Using Gallium Nanoparticles. Nano Lett. 2013, 13, 2837-2841.

16. Denisyuk, A. I.; Jonsson, F.; MacDonald, K. F.; Zheludev, N. I.; García De Abajo, F. J. Luminescence Readout of Nanoparticle Phase State. Appl. Phys. Lett. 2008, 92, 093112.

17. Soares, B. F.; Jonsson, F.; Zheludev, N. I. All-Optical PhaseChange Memory in a Single Gallium Nanoparticle. Phys. Rev. Lett. 2007, 98, 153905.

18. Soares, B. F.; MacDonald, K. F.; Zheludev, N. I. Resetting Single Nanoparticle Structural Phase with Nanosecond Pulses. Appl. Phys. Lett. 2007, 91, 043115.

19. Pochon, S.; MacDonald, K. F.; Knize, R. J.; Zheludev, N. I. Phase Coexistence in Gallium Nanoparticles Controlled by Electron Excitation. Phys. Rev. Lett. 2004, 92, 145702-1.

20. Soares, B. F.; MacDonald, K. F.; Fedotov, V. A.; Zheludev, N. I. Light-Induced Switching between Structural Forms with Different Optical Properties in a Single Gallium Nanoparticulate. Nano Lett. 2005, 5, 2104-2107.

21. Denisyuk, A. I.; MacDonald, K. F.; De Abajo, F. J. G.; Zheludev, N. I. Towards Femtojoule Nanoparticle Phase-Change Memory. Jpn. J. Appl. Phys. 2009, 48.

22. MacDonald, K. F.; Fedotov, V. A.; Zheludev, N. I. Optical Nonlinearity Resulting from a Light-Induced Structural Transition in Gallium Nanoparticles. Appl. Phys. Lett. 2003, 82, 1087-1089.

23. Yi, C.; Kim, T.-H.; Jiao, W.; Yang, Y.; Lazarides, A.; Hingerl, K.; Bruno, G.; Brown, A.; Losurdo, M. Evidence of Plasmonic Coupling in Gallium Nanoparticles/Graphene/SiC. Small 2012, 8, 2721-2730.

24. Sanz, J. M.; Ortiz, D.; Alcaraz de la Osa, R.; Saiz, J. M.; González, F.; Brown, A. S.; Losurdo, M.; Everitt, H. O.; Moreno, F. UV Plasmonic Behavior of Various Metal Nanoparticles in the Near- and Far-Field Regimes: Geometry and Substrate Effects. J. Phys. Chem. C 2013, 117, 1960619615.

25. Dogel, S.; Nattland, D.; Freyland, W. Complete Wetting Transitions at the Liquid-Vapor Interface of GalliumBismuth Alloys: Single-Wavelength and Spectroscopic Ellipsometry Studies. Phys. Rev. B: Condens. Matter Mater. Phys. 2005, 72, 085403.

26. Olmon, R. L.; Slovick, B.; Johnson, T. W.; Shelton, D.; Oh, S.-H.; Boreman, G. D.; Raschke, M. B. Optical Dielectric Function of Gold. Phys. Rev. B: Condens. Matter Mater. Phys. 2012, 86, 235147.

27. García de Abajo, F. J. Optical Excitations in Electron Microscopy. Rev. Mod. Phys. 2010, 82, 209-275.
28. Novotny, L.; Hecht, B. Principles of Nano-Optics; Cambridge University Press: Cambridge, U.K., 2006.

29. Bharadwaj, P.; Deutsch, B.; Novotny, L. Optical Antennas. Adv. Opt. Photonics 2009, 1, 438-483.

30. Wu, P. C.; Losurdo, M.; Kim, T.-H.; Choi, S.; Bruno, G.; Brown, A. S. In Situ Spectroscopic Ellipsometry to Monitor Surface Plasmon Resonant Group-III Metals Deposited by Molecular Beam Epitaxy. J. Vac. Sci. Technol., B 2007, 25, 1019.

31. Wu, P. C.; Losurdo, M.; Kim, T.-H.; Giangregorio, M.; Bruno, G.; Everitt, H. O.; Brown, A. S. Plasmonic Gallium Nanoparticles on Polar Semiconductors: Interplay between Nanoparticle Wetting, Localized Surface Plasmon Dynamics, and Interface Charge. Langmuir 2009, 25, 924-930.

32. Yang, Y.; Akozbek, N.; Kim, T.-H.; Sanz, J. M.; Moreno, F.; Losurdo, M.; Brown, A. S.; Everitt, H. O. Ultraviolet-Visible Plasmonic Properties of Gallium Nanoparticles Investigated by Variable-Angle Spectroscopic and Mueller Matrix Ellipsometry. ACS Photonics 2014, 1, 582-589.

33. Albella, P.; Garcia-Cueto, B.; González, F.; Moreno, F.; Wu, P. C.; Kim, T.-H.; Brown, A.; Yang, Y.; Everitt, H. O.; Videen, G. Shape Matters: Plasmonic Nanoparticle Shape Enhances Interaction with Dielectric Substrate. Nano Lett. 2011, 11, 3531-3537.

34. Kuttge, M.; Vesseur, E. J. R.; Koenderink, A. F.; Lezec, H. J.; Atwater, H. A.; García de Abajo, F. J.; Polman, A. Local Density of States, Spectrum, and Far-Field Interference of Surface Plasmon Polaritons Probed by Cathodoluminescence. Phys. Rev. B: Condens. Matter Mater. Phys. 2009, 79, 113405 .

35. García de Abajo, F. J.; Kociak, M. Probing the Photonic Local Density of States with Electron Energy Loss Spectroscopy. Phys. Rev. Lett. 2008, 100, 106804.

36. Sapienza, R.; Coenen, T.; Renger, J.; Kuttge, M.; van Hulst, N. F.; Polman, A. Deep-Subwavelength Imaging of the Modal Dispersion of Light. Nat. Mater. 2012, 11, 781-787.

37. Knight, M. W.; Liu, L.; Wang, Y.; Brown, L.; Mukherjee, S.; King, N. S.; Everitt, H. O.; Nordlander, P.; Halas, N. J. Aluminum Plasmonic Nanoantennas. Nano Lett. 2012, $12,6000-6004$.

38. Coenen, T.; Bernal Arango, F.; Koenderink, A. F.; Polman, A. Directional Emission from a Single Plasmonic Scatterer. Nat. Commun. 2014, 5, 3250.

39. Antosiewicz, T.J.;Apell, S.P.;Zäch, M.; Zorić, l.; Langhammer, C. Oscillatory Optical Response of an Amorphous TwoDimensional Array of Gold Nanoparticles. Phys. Rev. Lett. 2012, 109, 247401.

40. Nisoli, M.; Stagira, S.; De Silvestri, S.; Stella, A.; Tognini, P.; Cheyssac, P.; Kofman, R. Ultrafast Electronic Dynamics in Solid and Liquid Gallium Nanoparticles. Phys. Rev. Lett. 1997, 78, 3575-3578.

41. Lassiter, J. B.; McGuire, F.; Mock, J. J.; Ciracì, C.; Hill, R. T.; Wiley, B. J.; Chilkoti, A.; Smith, D. R. Plasmonic Waveguide Modes of Film-Coupled Metallic Nanocubes. Nano Lett. 2013, 13, 5866-5872.

42. Yamamoto, N.; Ohtani, S.; García de Abajo, F. J. Gap and Mie Plasmons in Individual Silver Nanospheres near a Silver Surface. Nano Lett. 2011, 11, 91-95.

43. Prodan, E.; Radloff, C.; Halas, N. J.; Nordlander, P. A Hybridization Model for the Plasmon Response of Complex Nanostructures. Science 2003, 302, 419-422.

44. Knight, M. W.; Wu, Y.; Lassiter, J. B.; Nordlander, P.; Halas, N. J. Substrates Matter: Influence of an Adjacent Dielectric on an Individual Plasmonic Nanoparticle. Nano Lett. 2009, 9, 2188-2192.

45. $\mathrm{Wu}, \mathrm{Y}$;; Nordlander, P. Finite-Difference Time-Domain Modeling of the Optical Properties of Nanoparticles near Dielectric Substrates. J. Phys. Chem. C 2010, 114, 73027307.

46. Bernal Arango, F.; Coenen, T.; Koenderink, A. F. Underpinning Hybridization Intuition for Complex Nanoantennas by Magnetoelectric Quadrupolar Polarizability Retrieval. ACS Photonics 2014, 1, 444-453.

47. Spinelli, P.; Macco, B.; Verschuuren, M. A.; Kessels, W. M. M.; Polman, A. $\mathrm{Al}_{2} \mathrm{O}_{3} / \mathrm{TiO}_{2}$ Nano-Pattern Antireflection 
Coating with Ultralow Surface Recombination. Appl. Phys. Lett. 2013, 102, 233902.

48. Spinelli, P.; Verschuuren, M. A; Polman, A. Broadband Omnidirectional Antireflection Coating Based on Subwavelength Surface Mie Resonators. Nat. Commun. 2012, 3, 692.

49. Rebien, M.; Henrion, W.; Hong, M.; Mannaerts, J. P.; Fleischer, M. Optical Properties of Gallium Oxide Thin Films. Appl. Phys. Lett. 2002, 81, 250-252.

50. Albella, P.; Moreno, F.; Saiz, J. M.; González, F. Surface Inspection by Monitoring Spectral Shifts of Localized Plasmon Resonances. Opt. Express 2008, 16, 12872.

51. Brandl, D. W.; Oubre, C.; Nordlander, P. Plasmon Hybridization in Nanoshell Dimers. J. Chem. Phys. 2005, 123, 024701.

52. Nordlander, P.; Oubre, C.; Prodan, E.; Li, K.; Stockman, M. I. Plasmon Hybridization in Nanoparticle Dimers. Nano Lett. 2004, 4, 899-903.

53. Lassiter, J. B.; Aizpurua, J.; Hernandez, L. I.; Brandl, D. W.; Romero, I.; Lal, S.; Hafner, J. H.; Nordlander, P.; Halas, N. J. Close Encounters between Two Nanoshells. Nano Lett. 2008, 8, 1212-1218.

54. Moreno, F.; Albella, P.; Nieto-Vesperinas, M. Analysis of the Spectral Behavior of Localized Plasmon Resonances in the Near- and Far-Field Regimes. Langmuir 2013, 29, 67156721.

55. Zuloaga, J.; Nordlander, P. On the Energy Shift between Near-Field and Far-Field Peak Intensities in Localized Plasmon Systems. Nano Lett. 2011, 11, 1280-1283.

56. Brown, L. V.; Sobhani, H.; Lassiter, J. B.; Nordlander, P.; Halas, N. J. Heterodimers: Plasmonic Properties of Mismatched Nanoparticle Pairs. ACS Nano 2010, 4, 819-832.

57. Mirsaleh-Kohan, N.; Iberi, V.; Simmons, P. D.; Bigelow, N. W.; Vaschillo, A.; Rowland, M. M.; Best, M. D.; Pennycook, S. J.; Masiello, D. J.; Guiton, B. S.; et al. Single-Molecule SurfaceEnhanced Raman Scattering: Can STEM/EELS Image Electromagnetic Hot Spots? J. Phys. Chem. Lett. 2012, 3, 23032309.

58. Kneipp, K.; Wang, Y.; Kneipp, H.; Perelman, L. T.; Itzkan, I.; Dasari, R. R.; Feld, M. S. Single Molecule Detection Using Surface-Enhanced Raman Scattering (SERS). Phys. Rev. Lett. 1997, 78, 1667-1670.

59. Rycenga, M.; Camargo, P. H. C.; Li, W.; Moran, C. H.; Xia, Y. Understanding the SERS Effects of Single Silver Nanoparticles and Their Dimers, One at a Time. J. Phys. Chem. Lett. 2010, 1, 696-703.

60. Li, W.; Camargo, P. H. C.; Lu, X.; Xia, Y. Dimers of Silver Nanospheres: Facile Synthesis and Their Use as Hot Spots for Surface-Enhanced Raman Scattering. Nano Lett. 2009, 9, 485-490.

61. Alonso-González, P.; Albella, P.; Schnell, M.; Chen, J.; Huth, F.; García-Etxarri, A.; Casanova, F.; Golmar, F.; Arzubiaga, L.; Hueso, L. E.; et al. Resolving the Electromagnetic Mechanism of Surface-Enhanced Light Scattering at Single Hot Spots. Nat. Commun. 2012, 3, 684.

62. Albella, P.; Alcaraz de la Osa, R.; Moreno, F.; Maier, S. A. Electric and Magnetic Field Enhancement with Ultralow Heat Radiation Dielectric Nanoantennas: Considerations for Surface-Enhanced Spectroscopies. ACS Photonics 2014, 1, 524-529.

63. Choi, S.; Kim, T.-H.; Everitt, H. O.; Brown, A.; Losurdo, M.; Bruno, G.; Moto, A. Kinetics of Gallium Adlayer Adsorption/ desorption on Polar and Nonpolar GaN Surfaces. J. Vac. Sci. Technol., B 2007, 25, 969.

64. Brenny, B. J. M.; Coenen, T.; Polman, A. Quantifying Coherent and Incoherent Cathodoluminescence in Semiconductors and Metals. J. Appl. Phys. 2014, 115, 244307.

65. Lumerical Solutions, Inc. http://www.lumerical.com.

66. Palik, E. D. Handbook of Optical Constants of Solids; Academic Press: San Diego, CA, 1998; Vol. 3.

67. Bruggeman, D. A. G. Berechnung Verschiedener Physikalischer Konstanten von Heterogenen Substanzen. I. Dielektrizitätskonstanten Und Leitfähigkeiten Der Mischkörper Aus Isotropen Substanzen. Ann. Phys. 1935, 416, 636664. 